\title{
Nivel de resiliencia del personal de salud frente a la pandemia por Covid-19
}

\section{Resilience level in health personnel facing the Covid-19 pandemic}

$\mathrm{DOI}$

https://doi.org/10.35434/rcmhnaaa.2021.14Sup1.1170

\section{RESUMEN}

Introducción: La resiliencia es un factor determinante para ayudar a afrontar alguna situación de estrés, por ello el objetivo de nuestro estudio, fue medir el nivel de resiliencia en el personal de salud en dos hospitales de la ciudad de Trujillo frente a la pandemia por Covid-19. El estudio: Se realizó un estudio descriptivo transversal con 106 trabajadores de salud, a quienes mediante una entrevista se les administró un cuestionario compuesto de 25 ítems. Hallazgos: Se encontró que la mayor parte de participantes fueron del sexo femenino $(67,9 \%$ vs $32,1 \%)$ y $40,6 \%$, personal médico. En ambos hospitales se halló un nivel alto de resiliencia, 95\% en el Hospital La Noria y $98 \%$ en EsSalud-La Esperanza. Conclusiones: El personal de salud presentó nivel alto de resiliencia frente a la pandemia del Covid-19.

Palabras Clave: Resiliencia; personal de salud; pandemias; infecciones por Coronavirus. (Fuente: DeCS-BIREME).

\section{ABSTRACT}

Background: Resilience is a determining factor in helping to cope with any stressful situation, therefore the objective of our study was to measure the resilience level in health personnel facing the COVID-19 pandemic at two hospitals in the city of Trujillo. The study: A crosssectional descriptive study was carried out with 106 health workers, who were administered a questionnaire consisting of 25 items through an interview. Findings: It was found that the majority of participants were female $(67.9 \%$ vs $32.1 \%)$ and $40.6 \%$, medical personnel. Conclusions: The health personnel presented a high resilience level facing the Covid-19 pandemic.

Keywords: Resilience; health personnel; pandemics; coronavirus infections (Source: DeCS-BIREME).
FILIACIÓN

1. Universidad César Vallejo, Trujillo, Perú.

a. Residente de Medicina Familiar y Comunitaria,

b. Médico de Medicina Familiar y Comunitaria,

ORCID

1. Kira Galina Becerra-Rodríguez 0000-0002-5856-797X

2. Linda Noheli Gamboa-Moreno 0000-0003-1723-2740

3. Yara Irene Lopez-Vergara 0000-0003-1940-1915

4. Evelyn Goicochea-Ríos 0000-0001-9994-9184

\section{CORRESPONDENCIA}

Linda Noheli Gamboa-Moreno

EMAIL

lindagm_16@hotmail.com

\section{CONFLICTOS DE INTERÉS}

Los autores niegan conflictos de interés.

\section{FINANCIAMIENTO}

Autofinanciamiento.

REVISIÓN DE PARES

Recibido: $15 / 04 / 2021$

Aceptado: 20/07/2021

\section{COMO CITAR}

Gamboa-Moreno, L., Becerra-Rodríguez, K., LópezVergara, Y., \& Goicochea-Ríos, E. Nivel de resiliencia del personal de salud frente a la pandemia por Covid-19. Revista Del Cuerpo Médico Hospital Nacional Almanzor Aguinaga Asenjo,2021, 14(Sup1), 49 - 54. https://doi.org/10.35434/rcmhnaaa.2021.14Sup1.1170
Esta obra está bajo una Licencia Creative Commons Atribución 4.0 Internacional.

Versión Impresa: ISSN: 2225-5109

Versión Electrónica: ISSN: 2227-473

Cross Ref. DOI: $10.35434 / \mathrm{rcmhnaaa}$

OJS: https://cmhnaaa.org.pe/ojs 


\section{INTRODUCCIÓN}

La enfermedad por Coronavirus (COVID-19) ha golpeado enormemente a nuestro país, produciendo que el sistema de salud colapse y perjudicando al personal de salud que se encuentra atendiendo directamente a personas con esta enfermedad. Debido a la gran demanda de pacientes, la misma enfermedad y el riesgo alto de contagio, el estrés ha aumentado, condicionando que los trabajadores usen mecanismos de defensa para no verse afectados en su salud mental; uno de ellos es la resiliencia, la cual es definida como la habilidad para luchar y afrontar la adversidad ${ }^{(1,2)}$. Esta palabra proviene del latín resilium, que significa "volver al estado inicial" "(3); Ortega $\mathrm{Z}^{(4)}$, refiere que la resiliencia es una habilidad que está presente en la vida del individuo y se va desarrollando conforme se van superando las adversidades, ayudando a aprender de ellas y preparando para afrontar las venideras ${ }^{(4)}$. La resiliencia surge de la combinación de los factores psicológicos (autoestima, manejo de las emociones, confianza de sí mismo) y factores sociales: familiares, comunicatorios que afecta su vida ${ }^{(3)}$.

A nivel mundial, la Organización Mundial de la Salud, hasta el 8 de abril de 2021, había notificado 132,485,386 casos confirmados de COVID-19, incluidas 2,875,672 muertes. A nivel de las Américas, 57,262,736 casos confirmados(5). En el Perú, hasta el 6 de abril del 2021 se reportaron 1,598,593 casos confirmados y 53,411 fallecidos, reportándose en el departamento La Libertad hasta la misma fecha 56,183 casos confirmados y 3,264 fallecidos ${ }^{(6)}$.

Narro-Cornelio $^{(7)}$, en un estudio realizado en diferentes hospitales de la provincia de Virú, halló que los jóvenes, adultos y adultos mayores constituyen el $92 \%$ del total de los casos, encontrándose un predominio de pacientes con COVID19 entre el rango de edad de 30 a 59 años con 159 casos $(51,9 \%)$. Asimismo, hubo un predominio de pacientes masculinos con respecto a los femeninos ( $57,4 \%$ vs $42,6 \%$ ).

La percepción de las personas sobre el coronavirus varía considerablemente; Iglesias-Osores(8) en un estudio realizado en 314 encuestados, la mayoría de ellos con estudios de pregrado, indica que el $71,30 \%$ señala que la principal vía de transmisión es aerógena, el 41,40\% indica que la letalidad es alta y $70,70 \%$, que existe tratamiento para la enfermedad.

Según Madariaga $L^{(9)}$, la resiliencia ayuda a satisfacer las necesidades del trabajador y disminuye el estrés y la frustración permitiendo crear una actitud positiva laboral; y para Caro $\mathrm{P}^{(10)}$, la resiliencia es un factor protector $y$ un instrumento útil para la promoción de la salud, y sería de gran utilidad incorporarla en programas de formación profesional.

O'Dowd $E^{(11)}$, realizaron entrevistas semiestructuradas para medir el estrés, el afrontamiento y la resiliencia en 68 médicos, de los cuales 39 eran mujeres y la media del tiempo de años trabajando fueron 7; encontrando en $18 \%$, la resiliencia como un rasgo y en $28,41 \%$, que la desarrollaron con tiempo y experiencia.

Lala $\mathrm{A}^{(12)}$, realizaron encuesta a 366 miembros del personal de
Unidades de Emergencia, haciendo uso de los instrumentos de la Escala de Estrés Percibido PSS-10 y el Cuestionario Breve COPE, encontrando la media de edad en 33 años, sin diferencias significativas entre sexos y un alto nivel de afrontamiento activo ( 3,10 puntos), aceptación ( 3,48 puntos) y planificación ( 2,89 puntos) en el grupo de médicos.

Fines $\mathrm{J}^{(13)}$, realizaron un estudio en 620 estudiantes de dos universidades donde buscaron conocer el nivel de resiliencia con la ansiedad y la salud; hallando que los varones presentaron puntuaciones más altas que las mujeres (media de 29,54 vs 25,38 ).

Ortunio $\mathrm{M}^{(14)}$, realizaron una encuesta a 33 trabajadores que laboraban más de 6 meses en una Unidad de Cuidados Intensivos Pediátricas en Venezuela, encontrando un mayor porcentaje de mujeres y personal de enfermería; además el $90 \%$ tenía más de dos años trabajando y el $45,5 \%$ presentó niveles medios de resiliencia.

Por último, Ruesga $\mathrm{J}\left({ }^{15)}\right.$, realizaron una correlación entre resiliencia y fatiga en 38 médicos residentes, encontrando un nivel de resiliencia muy desarrollado $22,5 \%$ y desarrollado en $77,50 \%$ y una relación moderada entre estas dos variables.

El impacto mundial del COVID-19 ha generado nuevos problemas relacionados con la parte psicológica; y en la salud, el incremento de pacientes con COVID-19, las defunciones diarias, los conflictos entre el paciente $\mathrm{y} / \mathrm{o}$ familiares del mismo y el personal de salud, el aumento de horas laborales sumado al miedo constante al contagio, ha producido en el personal de salud una sobrecarga de trabajo, estrés laboral y dificultad para lidiar con estas situaciones conllevando a una búsqueda frecuente de resiliencia frente a estas situaciones ${ }^{(16,17)}$.

La resiliencia puesta en práctica, permite tener niveles de estrés bajos frente a situaciones adversas y con ello, mejorar el clima laboral. Esta investigación nos permitirá contar con estudios locales sobre el tema y así, poder sugerir mejoras laborales que contribuyan a la salud mental de los trabajadores que se enfrentan día a día contra esta enfermedad, así como, estrategias que nos ayuden a sobrellevar situaciones de estrés laboral y, por ende, mejorar la calidad de vida y el ambiente laboral de todo personal de salud ${ }^{(18)}$. Es por tal motivo, que el objetivo del estudio fue establecer el nivel de resiliencia en el personal de salud de emergencia de dos hospitales de la ciudad de Trujillo frente a la pandemia del COVID-19 en el año 2020 y los objetivos específicos fueron identificar dicha variable según edad, género, ocupación y tiempo de servicio.

\section{ELESTUDIO}

Se realizó un estudio descriptivo de corte transversal realizado en 106 personal de salud del Hospital I ESSALUD-La Esperanza y del Hospital II-1 de Especialidades Básicas de la Noria, atendiendo pacientes COVID-19 durante marzo a agosto del 2020

La variable de interés para el estudio fue la resiliencia medida mediante el cuestionario de Agnild y Young ${ }^{(19)}$ y 
valorada en una escala ordinal.

Se consideraron como criterios de inclusión: personal de salud que trabajó en el área de emergencia del Hospital I de La Esperanza y Hospital II-1 de Especialidades Básicas de la Noria frente a la pandemia del COVID-19, que tuvo contacto directo en la atención de pacientes con COVID y que aceptó participar del estudio. Se excluyó al personal que estuvo ausente por cuarentena y aquellos que no respondieron todas las preguntas.

Se utilizó un formulario como recolección de datos, el mismo que estuvo conformado por variables generales como la edad, el sexo, la ocupación, el tiempo de servicio y el tiempo laborando en COVID; y variables especificas obtenidas mediante el Cuestionario de Resiliencia de Agnild y Young ${ }^{(19)}$. Este cuestionario consta de 25 ítems, cada uno con una escala Likert de 1 a 7 puntos, variando de 25 a 175 puntos, indicando que el mayor puntaje tiene una mayor resiliencia; además, evalúa 2 factores, el primero mide la competencia personal y está compuesto de 17 ítems; y el segundo, la aceptación de uno mismo y de la vida, el cual comprende el resto de ítems. Esta cuenta con una confiabilidad calculada por el coeficiente alfa de Cronbach de $0,89^{(20)}$.

Posteriormente, la información se procesó en una base de Excel para su posterior análisis estadístico utilizando porcentajes y la interpretación de las tablas, asimismo se utilizó el programa estadístico SPSS versión 18.

\section{RESULTADOS}

Se obtuvo una población total de 150 trabajadores de ambos hospitales, de los cuales 115 respondieron el cuestionario, siendo excluidos 9 participantes por encontrarse en cuarentena. Se encontró que 65 trabajadores fueron del Hospital II-1 de Especialidades Básicas de la Noria y 41, del Hospital de ESSALUD de La Esperanza.

Según el sexo, predominó el sexo femenino $(67,9 \%)$ sobre el masculino (32,1\%), encontrándose un alto nivel de resiliencia en las mujeres que en varones en ambos hospitales $(97,2 \%$ vs $94,1 \%$ ); además, el grupo en el que predominó la atención los pacientes con COVID-19, fueron en su mayoría médicos $(40,6 \%)$ y con respecto a la edad, se encontró que el personal que labora mayoritariamente están entre los 31 a 40 años $(36,8 \%)$ y no hubieron personas con más de 60 años en el desempeño de sus funciones (Tabla 1).

Los puntajes obtenidos al desarrollar el cuestionario de resiliencia de Agnild y Young, se encontraron entre 119 a 175 puntos, los cuales correspondieron al Hospital II-1 de La Noria, con una media general de $152,4 \pm 10$ y con una media para el hospital correspondiente de $152,3 \pm 10,6$; en cambio, los puntajes obtenidos para el Hospital ESSALUD La Esperanza, se encontró entre 126 a 174 puntos, con una media de $152,7 \pm 8,9$
Tabla 1. Características del personal de salud que atienden pacientes covid-19. Hospitales La Noria y ESSALUD-La Esperanza, 2020.

\begin{tabular}{|c|c|c|c|c|c|c|}
\hline \multirow{3}{*}{ Característica } & \multicolumn{4}{|c|}{ Hospital } & & \\
\hline & \multicolumn{2}{|c|}{ La Noria (65) } & \multicolumn{2}{|c|}{$\begin{array}{c}\text { ESSALUD La } \\
\text { Esperanza (41) }\end{array}$} & \multicolumn{2}{|c|}{ Total } \\
\hline & $\mathrm{N}$ & $(\%)$ & $\mathrm{N}$ & (\%) & $\mathrm{N}$ & $\%$ \\
\hline \multicolumn{7}{|l|}{ Sexo } \\
\hline Femenino & 47 & 72,3 & 25 & 61 & 72 & 67,9 \\
\hline Masculino & 18 & 27,7 & 16 & 39 & 34 & 32,1 \\
\hline \multicolumn{7}{|l|}{ Edad } \\
\hline 20 a 30 años & 12 & 18,5 & 8 & 19,5 & 20 & 18,9 \\
\hline 31 a 40 años & 17 & 26,2 & 21 & 51,2 & 38 & 35,9 \\
\hline 41 a 50 años & 29 & 44,6 & 5 & 12,2 & 34 & 32 \\
\hline$>50$ años & 7 & 10,8 & 7 & 17,1 & 14 & 13,2 \\
\hline \multicolumn{7}{|l|}{ Ocupación } \\
\hline Médico & 26 & 40 & 17 & 26,8 & 43 & 40,6 \\
\hline Enfermero & 12 & 18,5 & 11 & 41,5 & 23 & 21,7 \\
\hline Obstetra & 9 & 13,8 & 2 & 4,9 & 11 & 10,4 \\
\hline Personal de laboratorio & 7 & 10,8 & 5 & 12,2 & 12 & 11,3 \\
\hline Técnico en enfermería & 11 & 16,9 & 6 & 14,6 & 17 & 16 \\
\hline \multicolumn{7}{|l|}{ Tiempo de Servicio } \\
\hline 1 mes a 6 meses & 15 & 23,1 & 10 & 24,4 & 25 & 23,6 \\
\hline 7 meses a 1 año & 6 & 9,2 & 6 & 14,6 & 12 & 11,3 \\
\hline$>1$ año a 5 años & 11 & 16,9 & 14 & 34,1 & 25 & 23,6 \\
\hline$>5$ años & 33 & 50,8 & 11 & 26,8 & 44 & 41,5 \\
\hline \multicolumn{7}{|l|}{ Tiempo trabajando en COVID } \\
\hline 2 meses & 3 & 4,6 & 1 & 2,4 & 4 & 3,8 \\
\hline 3 meses & 7 & 10,8 & 5 & 12,2 & 12 & 11,3 \\
\hline 4 meses & 4 & 6,2 & 6 & 14,6 & 10 & 9,4 \\
\hline 5 meses & 11 & 16,9 & 5 & 12,2 & 16 & 15,1 \\
\hline 6 meses & 40 & 61,5 & 24 & 58,5 & 64 & 60,4 \\
\hline \multicolumn{7}{|l|}{ Contacto con Pacientes COVID } \\
\hline Solo Niños & 1 & 1,5 & 1 & 2,4 & 2 & 1,9 \\
\hline Solo Adultos & 7 & 10,8 & 16 & 39 & 23 & 21,7 \\
\hline Solo Gestantes & 10 & 15,4 & 2 & 4,9 & 12 & 11,3 \\
\hline Todas las edades & 47 & 72,3 & 22 & 53,6 & 69 & 65,1 \\
\hline
\end{tabular}

En ambos centros de salud se observa un nivel alto de resiliencia, siendo 95\% en el Hospital II-1 de La Noria y $98 \%$ en ESSALUD La Esperanza (Figura 1). Según la especialidad, los enfermeros, las obstetras y el personal de laboratorio tuvieron un alto nivel de resiliencia (100\%), comparado con los médicos (93\%) y técnicos en enfermería (94\%) (Figura 2).

En cuanto a las dimensiones del cuestionario de resiliencia, se pudo encontrar que en la dimensión Ecuanimidad, el 53,8\% del personal de salud de la Noria, predominó un alto nivel, mientras que en ESSALUD la Esperanza el 50,9\% predominó un nivel medio. Referente a la dimensión Sentirse bien sólo, en ambos centros de salud, predominó un nivel medio; con respecto a la Confianza en sí mismos y a la Perseverancia, la mayor parte del personal La Noria y Essalud, se encontró en un nivel alto. Finalmente, en la Satisfacción del personal, en ambos centros de salud se observa un nivel medio (Tabla 2 ). 


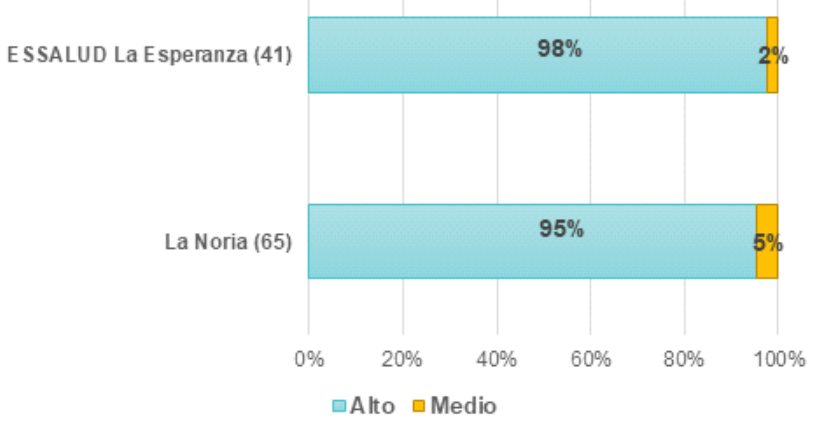

Figura 1

Nivel de resiliencia del personal de salud que atienden pacientes COVID-19. Hospitales La Noria y ESSALUD-La Esperanza, 2020.

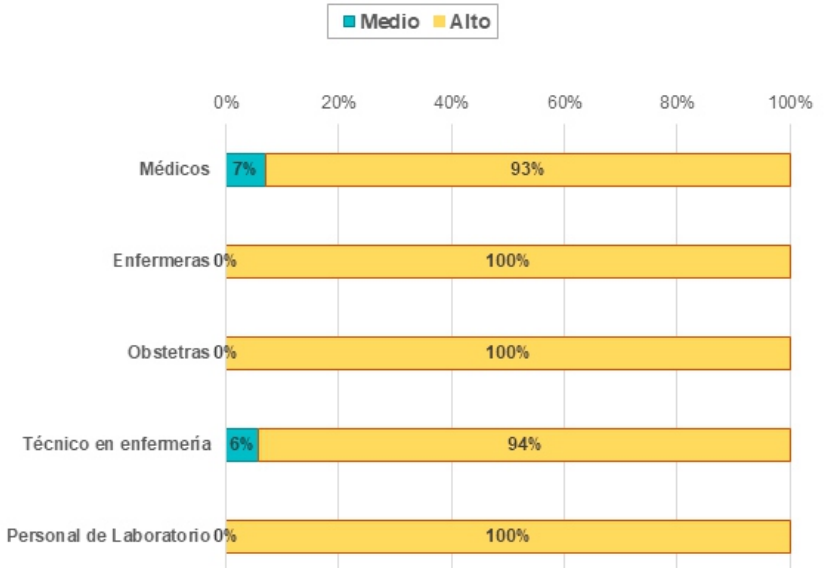

Figura 2

Nivel de resiliencia según la especialidad del personal de salud que atienden pacientes COVID-19. Hospitales La Noria y ESSALUD-La Esperanza, 2020.

Tabla 2. Nivel de las dimensiones de resiliencia del personal de salud que atienden pacientes covid-19. Hospitales La Noria y ESSALUD-La Esperanza, 2020.

\begin{tabular}{|c|c|c|c|c|c|c|c|}
\hline \multirow[b]{2}{*}{ Dimensiones } & \multirow[b]{2}{*}{ Nivel } & \multicolumn{2}{|c|}{ La Noria (65) } & \multicolumn{2}{|c|}{ ESSALUD La Esperanza (41) } & \multicolumn{2}{|c|}{ Total } \\
\hline & & $\mathrm{N}$ & $(\%)$ & $\mathrm{N}$ & (\%) & $\mathrm{N}$ & $\%$ \\
\hline \multirow[t]{2}{*}{ Ecuanimidad } & Medio & 30 & $46,2 \%$ & 24 & $58,5 \%$ & 54 & $50,9 \%$ \\
\hline & Alto & 35 & $53,8 \%$ & 16 & $39.00 \%$ & 51 & $48,1 \%$ \\
\hline Sentirse bien & Alto & 22 & $33,8 \%$ & 9 & $22.00 \%$ & 31 & $29,2 \%$ \\
\hline \multirow{2}{*}{ Confianza en sí mismo } & Medio & 30 & $46,2 \%$ & 18 & $43,9 \%$ & 48 & $45,3 \%$ \\
\hline & Alto & 35 & $53,8 \%$ & 23 & $56,1 \%$ & 58 & $54,7 \%$ \\
\hline \multirow{2}{*}{ Perseverancia } & Medio & 27 & $41,5 \%$ & 18 & $43,9 \%$ & 45 & $42,5 \%$ \\
\hline & Alto & 38 & $58,5 \%$ & 23 & $56,1 \%$ & 61 & $57,5 \%$ \\
\hline \multirow{3}{*}{ Satisfacción personal } & Bajo & 2 & $3,1 \%$ & 0 & $0,00 \%$ & 2 & $1,9 \%$ \\
\hline & Medio & 45 & $69,2 \%$ & 30 & $73,2 \%$ & 75 & $70,8 \%$ \\
\hline & Alto & 18 & $27,7 \%$ & 11 & $26,8 \%$ & 29 & $27,4 \%$ \\
\hline
\end{tabular}

\section{DISCUSIÓN}

Las circunstancias actuales han puesto de manifiesto la capacidad de adaptación del personal hospitalario, tales como la resiliencia, siendo ésta una habilidad que está presente en la vida del individuo y se va desarrollando conforme supera las adversidades, actuando como factor protector en la salud mental ${ }^{(4,10)}$.

El estudio encontró que el nivel de resiliencia del personal de salud en ambos hospitales fue alto, siendo $95 \%$ en la Noria y 98\% en ESSALUD La Esperanza. Estos resultados fueron más altos que los estudios realizados por Aranda $A^{(21)}$ en Trujillo, quien concluyó que los niveles de resiliencia en el personal de enfermería del servicio de emergencia del Hospital Víctor
Lazarte Echegaray-Essalud fue del $50 \%$ (nivel medio) y el realizado por Ortunio $\mathrm{M}^{(14)}$, quienes también hallaron un nivel medio de resiliencia en $45,5 \%$ de su población. Este hallazgo en nuestro estudio puede deberse a que el personal presentaba de 7 meses a menos en las labores de pacientes con Covid-19 y son hospitales que no veían pacientes críticos, ya que este tipo de pacientes eran evaluados y tratados en centros de mayor capacidad resolutiva y, por ende, no se encontraban con un nivel de estrés alto al tener pacientes hospitalizados y/o complicados como en otros hospitales.

En cuanto a la edad, se encontró entre los 30-40 años y el sexo femenino predominó en el estudio, guardando relación con el estudio de Ortunio $\mathrm{M}^{(14)}$, en la que la media de la edad fue 38,6 años y más del $80 \%$ era del sexo femenino. Sin embargo, el 
personal que predominó en nuestro estudio, fueron médicos a diferencia de Ortunio, que fueron enfermeras; esto puede deberse a que el personal que se encontró en primera línea y en horarios rotativos en atención a pacientes con Covid-19 fue el personal médico, entre médicos asistentes y residentes. Además, se encontró un alto nivel de resiliencia en el sexo femenino $(97,2 \%)$, a comparación de otros estudios $^{(22,23)}$ que encuentran valores bajos, debido a que la mujer también tiene que asumir el rol de crianza de los hijos y tiene menos tiempo de recreación; sin embargo, estos estudios tienen un pequeño tamaño muestral, por lo que no podrían ser tan significativos.

Por otro lado, los médicos tuvieron menor resiliencia en el estudio a comparación de otras especialidades, lo cual viene refrendado por el trabajo de $M c C a n^{(24)}$, quien encontró una media de $68,9 \%$ debido a que los médicos presentan un alto agotamiento y por ende un alto estrés traumático secundario.

Las dimensiones, Confianza en sí mismo y Perseverancia, presentaron los puntajes más altos en el personal de salud, este dato se correlaciona con el estudio hecho por Huamani ${ }^{(25)}$ en el Hospital Regional Honorio Delgado Espinoza de Arequipa, en el que se encontró a la Perseverancia como dimensión más afectada (50\%) en 50 enfermeras. En cambio, el estudio de Lala et al $^{(12)}$ haciendo uso del cuestionario COPE, se encontró que el Afrontamiento activo y la Planificación son los métodos usados por médicos para afrontar el estrés, mientras que el personal de enfermería, afrontar activamente y reinterpreta positivamente el problema. Extremera $\mathrm{N}^{(26)}$ hallan que la resiliencia se asocia a una mayor percepción de la autoeficacia.

Los niveles de resiliencia encontrados en trabajadores de salud de los Hospitales La Noria y Essalud-La Esperanza les permiten sentirse bien, con ecuanimidad y tener confianza en sí mismos, por lo que la resiliencia ayuda a satisfacer las necesidades del trabajador disminuyendo su estrés y la frustración creando una actitud laboral positiva. La realización de este trabajo brinda un aporte de información valiosa a profesionales y gestores para poder trabajar en áreas muy afectadas por el Covid-19 y así mejorar niveles de resiliencia en todo el personal de salud; además, se podría ver la posibilidad de hacer estudios en hospitales de diferente capacidad resolutiva para evaluar y comparar la resiliencia en grupos afectados a diversos niveles de estrés.

\section{REFERENCIAS BIBLIOGRÁFICAS}

1. Masten AS. Resilience in developing systems: Progress and promise as the fourth wave rises. Dev Psychopathol. 2007;19(3):921-30. doi: 10.1017/S0954579407000442.

2. Castillo JAG del, Castillo-López ÁG del, López-Sánchez C, Dias PC. Conceptualización teórica de la resiliencia psicosocial y su relación con la salud. Health and Addictions. 2016;16(1):59-68. doi:10.21134/haaj.v16i1.263.

3. Villalba-Quesada C. El concepto de resiliencia individual y familiar: Aplicaciones en la intervención social. Psy intervention [Internet]. 2003 [citado 27 oct 2020];12(3):283-99. Disponible en: https: / / www.redalyc.org/articulo.oa?id=179818049003.

4. González ZO, Llamozas BM. Concepto de resiliencia: Desde la diferenciación de otros constructos, escuelas y enfoques. Rev Cient Electron Cienc Hum [Internet]. 2018 [citado el 27 de octubre de
2020 ] ; $39\left(\begin{array}{ll}13 \\ 2\end{array}\right): 30-43$. D i s p o n ible e n : http://www.revistaorbis.org/pdf/39/art3.pdf.

5. WHO [Internet]. WHO Coronavirus (COVID-19) Dashboard; 2021 [cited 2021 apr 8]. Available from: https: / / covid19. who.int/.

6. Minsa. Sala Situacional COVID-19 Perú [Internet]. Lima: Ministerio de Salud del Perú; 2021 [citado el 8 de abril del 2021]. Disponible en: https://covid19.minsa.gob.pe/sala_situacional.asp.

7. Narro-Cornelio KM, Vásquez-Tirado GA. Características clínicoepidemiológicas en pacientes con diagnóstico covid-19. Red de salud Virú, marzo - mayo 2020. Rev cuerpo méd HNAAA. 2020;13(4):372-77. doi: 10.35434/rcmhnaaa.2020.134.772.

8. Iglesias-Osores S, Saavedra-Camacho J, Acosta-Quiroz J, CórdovaRojas L, Rafael-Heredia A. Percepción y conocimiento sobre COVID19: Una caracterización a través de encuestas. Rev cuerpo méd HNAAA. 2020;13(4):356-60. doi:10.35434/rcmhnaaa.2020.134.763.

9. Madariaga-Urriaga L. Resiliencia frente a problemas personales y el ámbito laboral en los trabajadores [Tesis]. Barrancabermeja: Universidad Cooperativa de Colombia; 2018 [citado 1 ago 2020]. D i s p o n i b l e

e n : https://repository.ucc.edu.co/bitstream/20.500.12494/6719/1/20 18 resiliencia ambito_laboral.pdf.

10. Caro-Alonso PA, Rodriguez-Martin B. Potencialidades de la resiliencia para los profesionales de Enfermería. Index Enferm [Internet]. 2018 [citado 1 ago 2020];27(1-2):42-6. Disponible en: http: //scielo.isciii.es/scielo.php?script=sci arttext\&pid=\$113212962018000100009 \&lng=es.

11. O'Dowd E, O'Connor P, Lydon S, Mongan O, Connolly F, Diskin C, et al. Stress, coping, and psychological resilience among physicians. BMC Health Serv Res. 2018; 18(1):730. doi: 10.1186/s12913-018-3541-8.

12. Lala Al, Sturzu LM, Picard JP, Druot F, Grama F, Bobirnac G. Coping behavior and risk and resilience stress factors in French regional emergency medicine unit workers: a cross-sectional survey. J Med Life [Internet]. 2016 [citado 1 ago 2020];9(4):363-8. Disponible en: https://pubmed.ncbi.nlm.nih.gov/27928439/.

13. Fínez Silva MJ, Morán Astorga MC. La resiliencia y su relación con salud y ansiedad en estudiantes españoles. Int J Dev Educ Psychol. 2015;1(1):409-16. doi:10.17060/ijodaep.2015.n1.v1.90.

14. Ortunio MS, Rodriguez A. Resiliencia en Trabajadores de la Salud de una Unidad de Cuidados Intensivos Pediátricos. Rev Ven Sal Pub [Internet]. 2019 [citado 27 oct 2020];7(1):27-33. Disponible en: https: //revistas.ucla.edu.ve/index.php/rvsp/article/view/2182.

15. Ruesga Pons JP, Carrasquero Carrasquero EE. Fatiga y resiliencia en el personal médico de los hospitales públicos. Multiciencias [Internet]. 2010 [citado 27 oct 2020];10:271-277. Disponible en: https: //www.redalyc.org/articulo.oa?id=90430360015.

16. Chesak SS, Perlman AI, Gill PR, Bhagra A. Strategies for Resiliency of Medical Staff During COVID-19. Mayo Clin Proc. 2020;95(9):S56-S59. doi:10.1016/j.mayocp.2020.07.002.

17. Greenberg N, Docherty M, Gnanapragasam S, Wessely S. Managing mental health challenges faced by healthcare workers during covid19 pandemic. BMJ. 2020;368:m1211. doi:10.1136/bmj.m1211.

18. García-Morán M, Gil-Lacruz $M$. El estrés en el ámbito de los profesionales de la salud. Persona. 2016;19:11-30. doi:10.26439/persona2016.n019.968.

19. Carina M, Andreu J. Validación psicométrica de la escala de Resiliencia (RS) en una muestra de adolescentes portugueses. Psicopat Clin Leg Foren [Internet]. 2011 [citado 27 oct $20020] ; 11(1): 51-65$. D i s p o n i ble e n : https://dialnet.unirioja.es/servlet/articulo?codigo=6383207

20. Salgado A. Métodos e instrumentos para medir la Resiliencia: Una alternativa peruana. LIBERABIT [Internet]. 2005 [citado 27 oct $2020]$; $11(1): 41-8$. D i s p o n i b l e e n : http: / / pepsic.bvsalud.org/scielo.php?script=sci_arttext\&pid=\$1729 $-48272005000100006$.

21. Aranda-Ruiz AB. Relación entre factores personales y resiliencia en enfermeras del servicio de emergencia del Hospital Víctor Lazarte Echegaray - ESSALUD. Repositorio Universidad Privada Antenor Orrego [Internet]. 2019 [citado 27 oct 2020]. Disponible en: https: / / repositorio.upao.edu.pe/handle/20.500.12759/5949.

22. Glasberg AL, Eriksson S, Norberg A. Burnout and 'stress of conscience' among healthcare personnel. J Adv Nurs. 2007;57(4):392-403. doi:10.1111/j.1365-2648.2007.04111.x.

23. Unrath M, Zeeb H, Letzel S, Claus M, Escobar L. Identification of possible risk factors for alcohol use disorders among general 
practitioners in Rhineland-Palatinate, Germany. Swiss Med Wkly. 2012;142:w13664. doi:10.4414/smw.2012.13664.

24. McCain RS, McKinley N, Dempster M, Campbell W, Kirk SJ. A study of the relationship between resilience, burnout and coping strategies in doctors. Postgrad Med J. 2017;0:1-5. Doi:10.1136/postgradmedj2016-134683.

25. Huamani-Huamani L, Mamani-Chinchercoma JV. Satisfacción laboral y capacidad de resiliencia en enfermeras de centro quirúrgico Hospital Regional Honorio Delgado Espinoza Arequipa 2017 [Tesis].
Arequipa: Universidad Nacional de San Agustín de Arequipa; 2018 [ C i t a d o 11 dic 2020 ]. Dis p on ible e n : http://repositorio.unsa.edu.pe/handle/UNSA/2735.

26. Extremera-Pacheco N, Durán-Durán MA, Rey L. Inteligencia emocional y su relación con los niveles de burnout, engagement y estrés en estudiantes universitarios. Rev Educ [Internet]. 2007 [citado 27 oct 2020];342:239-56. Disponible en: http://www.revistaeducacion.educacion.es/re342/re342_12.pdf. 